\title{
Investigation of Surfactant Efficiency Using Dissipative Particle Dynamics
}

\author{
Live Rekvig, ${ }^{, \dagger}$ Marieke Kranenburg, ${ }^{\ddagger}$ J ocelyne Vreede, ${ }^{\ddagger}$ Bjørn Hafskjold, ${ }^{\dagger}$ and \\ Berend Smit ${ }^{\ddagger}$
}

Department of Chemistry, Norwegian University of Science and Technology, N-7491 Trondheim, Norway, and Department of Chemical Engineering, University of Amsterdam, Nieuwe Achtergracht 166, 1018 WV Amsterdam, The Netherlands

Received April 15, 2003. In Final Form: J uly 9, 2003

\begin{abstract}
We have used dissipative particle dynamics (DPD) to simulate surfactant monolayers on the interface between oil and water. With a simple surfactant model, we investigate how variati ons in sizeand structure of surfactants influence their ability to reduce the interfacial tension. In particular, we studied the effect of branching of the hydrophobictail. We found that branched surfactants aremore efficient at theinterface than linear ones only if the head groups are sufficientl y hydrophilicto prevent themol ecul es from staggering. By combining DPD with a M onte Carlo method, we have imposed constant surfactant chemical potential and (normal) pressure in separate simulations of bulk and interface. From this, we can determine the bulk concentration needed to obtain a given interfacial tension. Wefound that higher concentrations of branched surfactants are required to obtain the samereduction of the interfacial tension. Wearguethat the stronger excluded volume interactions which make branched surfactants more efficient at the interface compared to their linear isomers at the same time make them less inclined to adsorb at the interface.
\end{abstract}

\section{Introduction}

Surfactants are of importance in many industrial applications. They adsorb on the interface between oil and water, lowering theinterfacial tension and promoting mixing. This is sometimes an undesired side effect, but usually the surfactants are added for this purpose. When choosing between the many surfactants available, both natural and artificially prepared, or designing new ones, one would often like surfactants which reduce the interfacial tension efficiently by adding as littlesurfactant as possible.

The amount of surfactants required to obtain a given interfacial tension reduction depends on several factors: The efficiency at the interface, the partitioning between bulk and interface, and the tendency to form micellar aggregates. Experimentally, it is common to distinguish between efficiency and effectiveness. ${ }^{1}$ The efficiency of a surfactant is defined as the negative logarithm of the surfactant concentration in bulk needed to reduce the interfacial tension by a given amount. In analogy with this, we define efficiency at the interface to reflect the interfacial densities (i.e., molecules per area) required to obtain a given interface effect. Surfactant effectiveness is defined as the maximum reduction of the interfacial tension a surfactant can produce, regardless of amount used.

The efficiency and effectiveness of a surfactant are related to its chemical structure. ${ }^{1}$ Many of thesurfactants prepared from petroleum feedstocks have branched hydrocarbon chains. Whereas, for example, the effect of increasing tail length on the interfacial tension is well-

* Corresponding author. E-mail: live.rekvig@phys.chem.ntnu.no. On leave at the Department of Chemical Engineering, University of Amsterdam.

${ }^{\dagger}$ Norwegian University of Science and Technology.

\# University of Amsterdam.

(1) Rosen, M. J. Surfactants and interfacial phenomena, 2nd ed.; Wiley: New York, 1989. known and understood (Traube's rule), 2,3 thereareno such clear rules concerning the effect of branching the hydrophobic tail. Although Rosen ${ }^{1}$ summarizes that linear surfactants aremore efficient than branched isomers, both positive $\mathrm{e}^{4,5}$ and negative $\mathrm{e}^{6,7}$ effects of branching on surfactant efficiency are reported in the literature and the effect is still not fully understood.

The degree of branching can vary from the doublechain obtained by moving the hydrophilic head group al ong the chain, to Guerbet branched surfactants, wherethe second chain is attached to the al pha or beta carbon of the main chain, to highly branched surfactants, where methyl groups are attached at several sites along the single or doublechains. For example, Varadaraj et al. investigated linear, Guerbet branched, and highly branched surfactants. 4,8,9 They found that for ethoxy sul fate surfactants, both efficiency and effectiveness increase with Guerbet branching on an oil/water interface (although less pronounced than on an air/water interface), and they attribute this tothe efficient packing at the interface. ${ }^{4}$ Wormuth et al . found Guerbet branched surfactants to beless efficient in mixing oil and water than the linear surfactants of the same number of carbon atoms and with head groups of either five ethoxy groups or a sulfate head group. ${ }^{6}$ They ascribed this to less efficient packing and more flexible monolayers. Further branching was found to lower the

(2) Smit, B. Phys. Rev. A 1988, 37, 3431-3433.

(3) Smit, B.; Schlijper, A. G.; Rupert, L. A. M.; van Os, N. M.J . Phys. Chem. 1990, 94, 6933-6935.

(4) Varadaraj, R.; Bock, J .; Valint, P., J r.; Zushma, S.; Thomas, R. J . Phys. Chem. 1991, 95, 1671-1676.

(5) Pitt, A. R.; Morley, S. D.; Burbidge, N. J .; Quickenden, E. L. Colloids Surf., A 1996, 114, 321-335.

(6) Wormuth, K. R.; Zushma, S. Langmuir 1991, 7, 2048-2053.

(7) Aspée, A.; Lissi, E. J . Colloid Interface Sci. 1996, 178, 298-302.

(8) Varadaraj, R.;Bock, J .;Zushma, S.; Brons, N.; Colletti, T.J .Colloid Interface Sci. 1991, 147, 387-395.

(9) Varadaraj, R.; Bock, J .; Geissler, P.; Zushma, S.; Brons, N.; Colletti, T. J . Colloid Interface Sci. 1991, 147, 396-402. 
efficiency significantly $y^{8,6,10}$ due to a less compact monolayer. Aspée and Lissi, investigating al cohol s of different topol ogy and chain lengths, concluded that the branched isomers have a larger tendency to adsorb at the interface at low surface pressures, whereas linear surfactants are more efficient at higher surface pressure. ${ }^{7}$

Although the interfacial tension measurements are rel atively straightforward, it is difficult to obtain detailed information on the behavior of the amphiphilic molecules and their concentration at the interface as this requires expensive neutron scattering experiments. ${ }^{11}$ Therefore, molecular simulations are an attractive alternative to provide additional information on distributions and ordering of theamphiphiles, enhancing our understanding of surfactant efficiency at the interface. In the process of designing or choosing new surfactants, it is valuable to know whether a surfactant is more efficient than another because of a greater tendency to adsorb on the interface or because it is better at reducing the interfacial tension at the interface. In the simulations reported here, we investigate both effects separately.

Beginning in the 1990s, modern computers have made it possible to study surfactant efficiency at the interface using simulations at themol ecular scale, such as mol ecular dynamics (MD). These give very detailed information on the monolayers formed, and the effect of for example increased chain length has been successfully studied. ${ }^{3}$ However, the time scales accessible to ordinary MD simulations are too short to observe diffusion to the interface and formation of micelles. Spontaneous formation of a micelle takes a few nanoseconds and could be doneusing fully atomisticmodels. ${ }^{12}$ Micellereorganization and diffusion between interface, micelle, and bulk are likely to be slower. Molecular simulations can thus presently only give information on the efficiency at the interface.

An alternative approach is to simulate an oil/water/ surfactant system at a mesoscopic level, for example, lumping two or threewater mol ecules intoa singlecoarsegrained particle. By coarse graining the interactions between the atoms, we can access longer length and time scales. The dissipative particle dynamics (DPD) technique ${ }^{13,14}$ is ideal tosimulatesuch a coarse-grained system and is what we opted for here. Although less detailed than $M D$, it still enables a systematic study of the effect of surfactant structure on the interfacial tension. With a simple model, we can mimic variations in chemical structure such as branching or increasing the tail length. By varying the interaction between the dissipative particles, the effect of, for example, salt addition can be studied. In particular, we investigate how efficiency of surfactants is related to ordering at the interface.

\section{Model and Simulation Details}

2.1. Dissi pative Particle Dynamics. In DPD, conservative, random, and dissipativeforces act between two particles $\mathrm{i}$ and $\mathrm{j}$ which are a distance $\mathrm{r}_{\mathrm{ij}}$ apart.

$$
\mathbf{F}_{\mathrm{ij}}=\mathbf{F}_{\mathrm{ij}}^{\mathrm{C}}\left(\mathrm{r}_{\mathrm{ij}}\right)+\mathbf{F}_{\mathrm{ij}}^{\mathrm{R}}\left(\mathrm{r}_{\mathrm{ij}}\right)+\mathbf{F}_{\mathrm{ij}}^{\mathrm{D}}\left(\mathrm{r}_{\mathrm{ij}}\right)
$$

(10) Ringard-Lefebvre, C.; Bochot, A.; Memisoglu, E.; Charon, D.; Duchene, D.; Baszkin, A. Colloids Surf., B 2002, 25, 109-117.

(11) Lu, J . R.; Thomas, R. K.; Penfold, J . Adv. Colloid Interface Sci. 2000, 84, 143-304.

(12) Marrink, S. J .; Tieleman, D. P.; Mark, A. E. J . Phys. Chem. B 2000, 104, 12165-12173.

(13) Hoogerbrugge, P. J .; Koelman, J . M. V. A. Europhys. Lett. 1992 19, $155-160$

(14) Español, P.; Warren, P. B. Europhys. Lett. 1995, 30, 191-196.

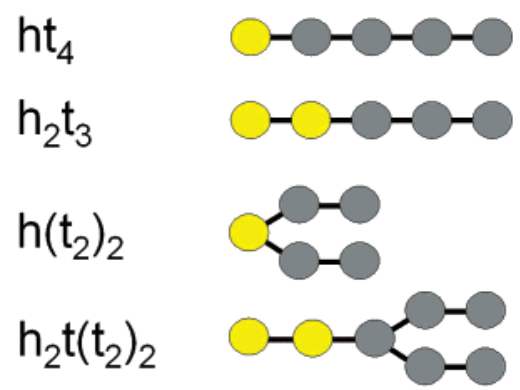

Figure 1. Some of the model surfactants investigated in this study and the nomenclature used. The yellow particles are hydrophilic beads (h), and the gray particles are hydrophobic tails (t).

where the forces are of the form

$$
\begin{gathered}
\mathbf{F}_{\mathrm{ij}}^{\mathrm{C}}=\mathrm{a}_{\mathrm{ij}} \mathrm{w}^{\mathrm{C}}\left(\mathrm{r}_{\mathrm{ij}}\right) \hat{\mathbf{r}}_{\mathrm{ij}} \\
\mathbf{F}_{\mathrm{ij}}^{\mathrm{R}}=\sigma \mathrm{w}^{\mathrm{R}}\left(\mathrm{r}_{\mathrm{ij}}\right) \theta_{\mathrm{ij}} \hat{\mathbf{r}}_{\mathrm{ij}} \\
\mathbf{F}_{\mathrm{ij}}^{\mathrm{D}}=-\eta \mathrm{w}^{\mathrm{D}}\left(\mathrm{r}_{\mathrm{ij}}\right)\left(\hat{\mathbf{r}}_{\mathrm{ij}} \cdot \mathbf{v}_{\mathrm{ij}}\right) \hat{\mathbf{r}}_{\mathrm{ij}}
\end{gathered}
$$

Here, $\mathbf{v}_{\mathrm{ij}}$ is the velocity difference for the two particles, and $\hat{\mathbf{r}}_{\text {ij }}$ is the unit vector pointing from particl ei to particle j. $\theta$ is a random number between 0 and $1 . \mathrm{a}_{\mathrm{ij}}, \sigma$, and $\eta$ determinetheamplitude of theconservative, random, and dissipative forces, respectively, while the w's are weight functions. To obey the fluctuation-dissipation theorem, we must have $w^{D}=\left(w^{R}\right)^{2}$, and the system temperature will follow from the relation between $\sigma$ and $\eta: \sigma^{2} / \eta=2 \mathrm{kT}$. We use thesameintegration algorithm, weight functions, and parameters as Groot and Warren:15

$$
w^{C}(r)=w^{R}(r)=\sqrt{w^{D}(r)}=w(r)
$$

where

$$
w(r)= \begin{cases}1-\frac{r}{r_{c}} & \text { for } r<r_{c} \\ 0 & \text { for } r \geq r_{c}\end{cases}
$$

Throughout this paper we use reduced units. $r_{c}$ is the unit of length, kT (the temperature of the thermostat) is the unit of energy, and the mass unit is the mass of a DPD bead. In these units, $\sigma=3.0$ and $\eta=4.5$.

2.2. Model. We use a coarse-grained approach where one DPD particle represents a group of atoms or a liquid volume. Water beads, oil beads, head groups, and tail groups are denoted by w, o, h, and t, respectively. Thetail beads are identical to the oil beads. Some of the model surfactants investigated are shown in Figure 1. A surfactant consists of head groups and tail groups connected by harmonic springs:

$$
\mathbf{F}_{\mathrm{ij}}^{\text {Bond }}=-\mathrm{k}_{\mathrm{s}}\left(\mathrm{r}_{\mathrm{ij}}-\mathrm{r}_{0}\right) \hat{\mathbf{r}}_{\mathrm{ij}}
$$

We choose $k_{\mathrm{s}}=100$ and $r_{0}=0.7$. Water and oil are represented by a single bead for simplicity. Natural oil, however, is composed of hydrocarbon chains. To investigate if the results depend on the oil chain length, we performed some simulations for two types of surfactants at different oil chain lengths. Since these simulations do not show qualitativedifferences, wefocus in theremainder

(15) Groot, R. D.; Warren, P. B. J . Chem. Phys. 1997, 107, 4423- 


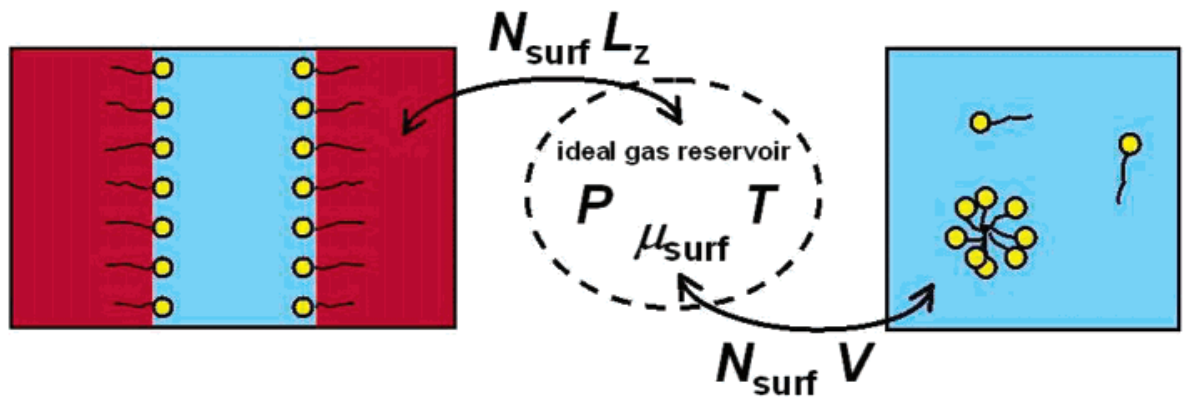

Figure 2. Illustration showing the system containingtheinterface (left) and the bulk system (right). They arecoupled to a reservoir (middle) with a constant surfactant chemical potential, pressure, and temperature. Both systems can exchange surfactants, heat, and volume with the reservoir. In the interfacial system $\mathrm{N}_{\text {water, }} \mathrm{N}_{\text {oil }}$, and $\mathrm{A}$ are kept constant; in the bulk $\mathrm{N}_{\text {water }}$ is fixed.

of this work on oil molecules that are represented by a single bead.

In some simulations, we study the effect of changes in the repulsion parameters $a_{i j}$ and mention their values explicitly. For the rest, we use the repulsion parameters shown in Table 1. These are taken from Groot, ${ }^{16}$ except that we used $\mathrm{a}_{\mathrm{o}}=25$ instead of 15 . This change has been made to obtain similar bulk densities in the oil and in the water phase.

2.3. Constant $\mu_{\text {surf }} \mathbf{P}_{\perp} \mathbf{T}$ Conditions. Experimentally, it is well-known that even if the oil/water interface is saturated with surfactant, theconcentration of surfactant in bulk can bevery small. Onether eforeneeds tosimulate a very largesystem to obtain a few surfactants in the bulk phase. Here we avoid these difficulties by simulating the setup shown in Figure 2. This setup is comprised of two systems: an inhomogeneous system which contains oil, water, and surfactants and a homogeneous system with water and surfactants only. The inhomogeneous system is used to determine the surfactant concentration at the interface and the interfacial tension. The homogeneous system is used to compute the bulk concentration of surfactants in the water phase. To ensure that the two systems arein equilibrium, both arecoupled toa reservoir that imposes the (normal) pressure, temperature, and chemical potential of the surfactants.

In the simulations, this is achieved by combining DPD with a Monte Carloscheme. ${ }^{17}$ Theinhomogeneous system consists of a fixed number of water particles $\mathrm{N}_{\text {water }}$ and oil particles $\mathrm{N}_{\text {oil }}$ and a fixed area $\mathrm{A}$. The box length and the number of surfactants in thesystemarevaried by imposing $\mu_{\text {surf }}$ and the normal pressure $\mathrm{P}_{\perp}$. In a separatesimulation, we determine the bulk concentration c culk corresponding to the same $\mu_{\text {surf. }}$ Here, $\mathrm{N}_{\text {water }}$ is kept constant and $\mathrm{P}$ and $\mu_{\text {surf }}$ are imposed.

In a one-component system, imposing $\mu, \mathrm{P}$, and $\mathrm{T}$ yields an unbound system since no extensive variables are specified. There is an infinite number of combinations of $\mathrm{N}$ and $\mathrm{V}$ that give the equilibrium density. When this density is obtained, the rest of the simulation will be a random walk in system size with $\mathrm{N}$ and $\mathrm{V}$ growing toward infinity. I n our systems, thenumber of particles of at least one component is fixed; therefore, the systems will be bound as long as the imposed $\mu_{\text {surf }}$ is sufficiently low such that a separate surfactant phase is avoided.

2.3.1. The Henry Regime. When the surfactant concentration in bulk is sufficiently low such that the

(16) Groot, R. D. Langmuir 2000, 16, 7493-7502.

(17) Willemsen, S. M.; Vlugt, T. J . H.; Hoefsloot, H. C. J .; Smit, B. J. Comput. Phys. 1998, 147, 507-517. surfactants do not interact, cbulk can be estimated from Henry's law,

$$
c_{\text {bulk }}=\frac{N_{\text {surf }}}{V}=K_{H} P_{\text {surf }}
$$

The Henry coefficient $\mathrm{K}_{H}$ is related to the excess chemical potential as

$$
\mathrm{K}_{\mathrm{H}}=\beta \exp \left(-\beta \mu_{\text {surf }}^{\mathrm{ex}}\right)
$$

$\mathrm{P}_{\text {surf }}$ is the partial pressure of a surfactant in theideal gas reservoir, which is directly related tothereservoir chemical potential (see Figure 2)

$$
\beta \mu_{\text {surf }}=\beta \mu_{\text {id.gas }}^{0}+\operatorname{In}\left(\beta \mathrm{P}_{\text {surf }}\right)
$$

Combining eqs 8-10 gives the expression which relates the imposed chemical potential, $\mu_{\text {surf }}$, to the concentration in the bulk water in the Henry regime:

$$
c_{\text {bulk }}=\exp \left[-\beta \mu_{\text {surf }}^{\text {ex }}\right] \exp \left[\beta\left(\mu_{\text {surf }}-\mu_{\text {id.gas }}^{0}\right)\right]
$$

$\mu_{\text {surf }}^{\mathrm{ex}}$ is calculated using the Widom insertion method:18

$$
\beta \mu^{\mathrm{ex}}=-\ln \int \mathrm{ds}^{\mathrm{N}+1}\langle\exp (-\beta \Delta \mathrm{U})\rangle_{\mathrm{N}}
$$

where $\Delta \mathrm{U}$ is the energy associated with the insertion of a test molecule into the $\mathrm{N}$-particle system.

2.3.2. Monte Carlo Algorithm. For the bulk system, simulations under constant $\mu_{\text {surf }}, N_{w}, P, T$ conditions involve three different types of Monte Carlo moves: (1) a series of DPD steps, (2) a change of volume, and (3) addition or removal of surfactants.

These are done independently (the volume is kept constant during an attempt to add or remove particles, and vice versa), which implies that the standard acceptancerules ${ }^{18}$ for theisobaric-isothermal ensembleand the grand canonical ensemble can be used, respectively.

In the inhomogeneous system, the interfacial area is kept constant and only the box length is varied in order to impose a normal pressure. Let o denote the old and $n$ the new configuration. As for the homogeneous system, the volume change $\Delta \mathrm{V}$ is drawn from a uniform distribu-

(18) Frenkel, D.; Smit, B. Understanding Molecular Simulations: From Al gorithms to Applications, 2nd ed.; Academic Press: San Diego, 2002. 

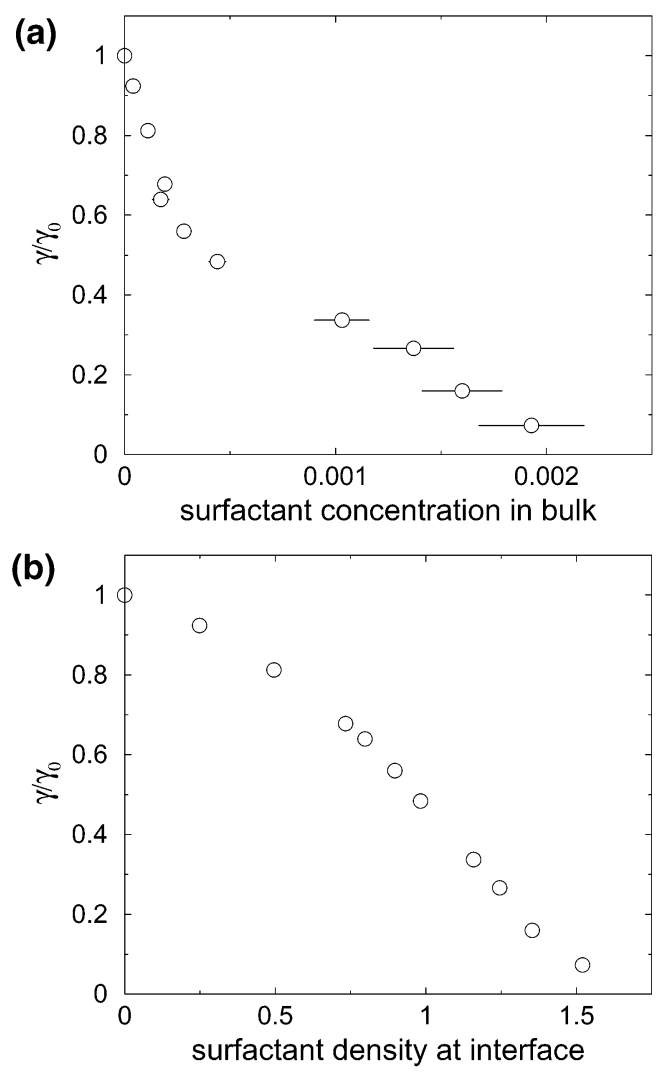

Figure 3. Normalized interfacial tension versus (a) surfactant concentration in bulk and (b) surfactant density at theinterface, for the ht surfactant.

tion and the acceptance rules remain thesame. However, the new box lengths in this system are

$$
\begin{gathered}
\mathrm{L}_{\mathrm{x}, \mathrm{n}}=\mathrm{L}_{\mathrm{x}, \mathrm{o}} \\
\mathrm{L}_{\mathrm{y}, \mathrm{n}}=\mathrm{L}_{\mathrm{y}, \mathrm{o}} \\
\mathrm{L}_{\mathrm{z}, \mathrm{n}}=\mathrm{L}_{\mathrm{z}, \mathrm{o}}\left(\frac{\mathrm{V}_{\mathrm{n}}}{\mathrm{V}_{\mathrm{o}}}\right)
\end{gathered}
$$

instead of

$$
\mathrm{L}_{\mathrm{n}}=\mathrm{L}_{\mathrm{o}}\left(\frac{\mathrm{V}_{\mathrm{n}}}{\mathrm{V}_{\mathrm{o}}}\right)^{1 / 3}
$$

for the bulk system.

2.3.3. Demonstration of the Method: A Simple Surfactant. We demonstrate the method by applying it to thesimple dumbbell surfactant ht. Figure 3 shows how the interfacial tension is reduced with increasing surfactant concentration in the bulk (a) and at the interface (b). The interfacial tension was calculated by dividing the simulation box into 100 slabs parallel to theinterfaceand calculating the pressure tensor in each slab: ${ }^{2}$

$$
\gamma=\frac{1}{2} \int_{0}^{L z}\left[p_{z z}(z)-\frac{1}{2}\left(p_{x x}(z)+p_{y y}(z)\right)\right] d z
$$

The interfacial tension without surfactants present, $\gamma_{0}$, is $3.45 \pm 0.03$.

To determine the surfactant density at the interface, we investigate the density profiles. An example is shown in Figure 4a. The average numbers of water, oil, tail, and head beads per volume unit are pl otted across the box. In this case, all thesurfactants in thebox areat theinterfaces,
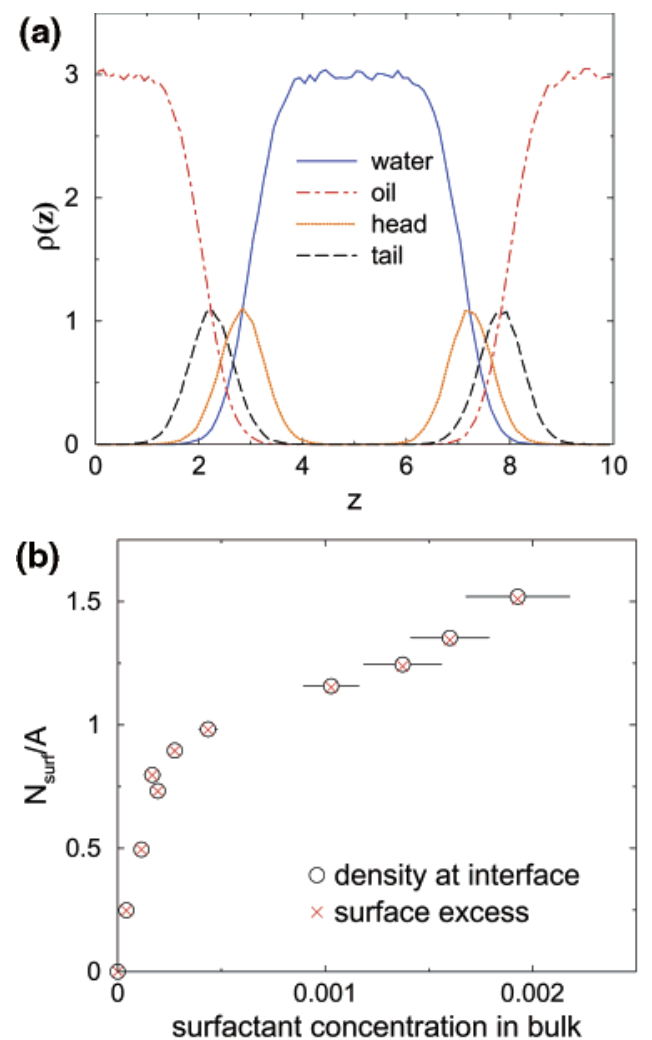

Figure4. (a) Density profiles at an average interfacial density of 1.15. (b) Surfactant density at theinterfaceand surfaceexcess versus surfactant concentration in the bulk.

and we define the density at the interface as the average number of surfactants in the system divided by twice the box area. Figure 4b shows the surfactant density at the interface versus the surfactant concentration in the bulk. The excess adsorption of surfactants, $\Gamma$, is also shown. As the surfactant is symmetric in oil and water and the two bulk phases have identical properties, $\Gamma$ is independent of the location of the interface and can be calculated from

$$
\Gamma=\frac{1}{2} \int_{0}^{L_{z}}\left[\rho_{\text {surf }}(z)-C_{\text {bulk }}\right] d z
$$

$\Gamma$ almost coincides with the surfactant density at the interface because the bulk concentration is very low.

Figures $3 a$ and $4 b$ both show an abrupt change in interfacial tension and interfacial density curves, respectively, at a specific bulk concentration. The surfactants in the bulk start to interact with each other only at sufficiently high imposed chemical potentials. This corresponds toa bulk concentration of ca. $2 \times 10^{-4}$ surfactants per volume unit and a $50 \%$ reduction of the interfacial tension. At higher concentrations, the surfactant density at theinterfaceincreases slower because moresurfactants go into micelles rather than to the interface. This causes theabrupt changein the $\gamma\left(c_{\text {surf }}\right)$ curve. For higher chemical potentials, the surfactants form a separate phase. I $n$ this phase, the fixed number of water particles does not limit thetotal amount of surfactants. Therefore, the box volume and the number of surfactants perform a random walk while the equilibrium mass density is maintained, that is, the system size grows toward infinity. This limits our method to concentrations up to just beyond the critical micelle (or aggregation) concentration.

2.4. Configurational-Biased Monte Carlofor Chain Molecules. Our method to compute the bulk densities relies on the successful insertion and deletion of the 
surfactant molecules. As we have shown in the previous section, for dumbbell surfactants this can be achieved using conventional Monte Carlo simulations. F or longer chain molecules, however, the probability to successfully insert a molecule is prohibitively small. To improve the acceptance ratio, we use configurational-biased Monte Carlo (CBMC). ${ }^{19,20}$ The idea of CBMC is to grow the chain segments one by one and, for each bead, choose an energetically favorable position.

The energy associated with each bead is separated into internal and external contributions, denoted $U^{\text {int }}$ and $U$ ext, respectively. A natural choice for our model is to let $U^{\text {int }}$ account for the harmonic bond potential and let $U$ ext contain the repulsive potential. A CBMC simulation involves the generation of trial conformations by growing a chain bead by bead and the computation of the Rosenbluth factor, which is needed to correct for the bias. The procedure to generate a trial insertion is as follows:

1. For the first monomer, generate $f$ trial positions at random and for each position i cal culate the energy $U$ ext. $(1, \mathrm{i})$ and the factor

$$
\mathrm{w}_{1}=\sum_{\mathrm{i}=1}^{\mathrm{f}} \exp \left[-\beta \mathbf{U}^{\mathrm{ext}}(1, \mathrm{i})\right]
$$

One of the trial positions is chosen with probability

$$
p_{1}^{\text {ext }}(i)=\frac{\exp \left[-\beta \cup^{e x t}(1, i)\right]}{w_{1}}
$$

2. To place the next segment $k, g$ trial positions are generated satisfying the probability distribution

$$
\mathrm{p}_{\mathrm{k}}^{\mathrm{int}}(\mathrm{i}) \propto \exp \left[-\beta \mathrm{U}^{\mathrm{int}}(\mathrm{k}, \mathrm{i})\right]
$$

where $U^{\text {int }}(k, i)$ is the internal interactions of monomer $k$ in the ith trial position with the already placed $k-1$ monomer(s). The factor

$$
\mathbf{w}_{k}=\sum_{i=1}^{g} \exp \left[-\beta \mathbf{U}^{e x t}(k, i)\right]
$$

is calculated where $U^{\text {ext }}(k, i)$ includes all external interactions between monomer $\mathrm{k}$ and the previously placed $\mathrm{k}-$ 1 monomers of the same chain plus all other molecules. One of the g trial positions is chosen with the probability

$$
p_{k}^{\text {ext }}(i)=\frac{\exp \left[-\beta U^{e x t}(k, i)\right]}{w_{k}}
$$

3. Step 2 is repeated until all $K$ segments of themolecule are grown. Then its Rosenbluth factor $W^{\text {ext }}$ is calculated as

$$
W^{e x t}=\prod_{k=1}^{K} w_{k}
$$

For removal of a chain, the following modification to the algorithm applies: Only $g-1$ trial positions are generated for each monomer ( $\mathrm{f}-1$ for the first monomer),

(19) Siepmann, J . I.; Frenkel, D. Mol. Phys. 1992, 75, 59-70

(20) Frenkel, D.; Mooij, G. C. A. M.; Smit, B.J . Phys.: Condens. Matter $1992,4,3053-3076$ the first being simply the present position of the bead. The $w_{k}$ 's are

$$
\begin{aligned}
& \mathrm{w}_{1}=\exp \left[-\beta \mathrm{U}^{\mathrm{ext}}(1, \text { old })\right]+\sum_{\mathrm{i}=2}^{\mathrm{f}} \exp \left[-\beta \mathrm{U}^{\mathrm{ext}}(1, \mathrm{i})\right] \\
& \mathrm{w}_{\mathrm{k}}=\exp \left[-\beta \mathrm{U}^{\mathrm{ext}}(\mathrm{k}, \text { old })\right]+\sum_{\mathrm{i}=2}^{\mathrm{g}} \exp \left[-\beta \mathrm{U}^{\mathrm{ext}}(\mathrm{k}, \mathrm{i})\right]
\end{aligned}
$$

and $W^{\text {ext }}$ is as defined above.

The acceptance rules become

$$
\operatorname{acc}\left(N_{\text {surf }} \rightarrow N_{\text {surf }}+1\right)=\min \left[1, \frac{\mathrm{Vq}(T)}{N_{\text {surf }}+1} \exp (\beta \mu) W^{\text {ext }}\right]
$$

to add a molecule and

$$
\operatorname{acc}\left(N_{\text {surf }} \rightarrow N_{\text {surf }}-1\right)=\min \left[1, \frac{N_{\text {surf }} \exp (-\beta \mu)}{q(T) V} \frac{1}{W^{\text {ext }}}\right]
$$

toremovea molecule, whereq $(T)$ is thekinetic contribution to the partition function, that is,

$$
-\mathrm{q}(\mathrm{T})=\beta \mu_{\mathrm{id} . \mathrm{gas}}-\beta \mu_{\mathrm{intra}}^{0}-\beta \ln \left(\frac{\mathrm{N}_{\text {surf }}}{\mathrm{V}}\right)
$$

CBMC can also be used to compute the excess chemical potential more efficiently. TheWidom insertions are done using the CBM C scheme, and eq 12 becomes, in terms of Rosenbluth factors,

$$
\beta \mu^{\mathrm{ex}}=-\ln \frac{\left\langle\mathrm{W}^{\mathrm{ext}}\right\rangle}{\left\langle\mathrm{W}_{\mathrm{id} . \text { gas }}^{\mathrm{ext}}\right\rangle}
$$

where $W_{\text {id.gas }}^{\text {ext }}$ is the Rosenbluth factor of an ideal gas, that is, accounting for theintramolecular external interactions of an ideal chain in the ideal gas phase. We use the same reference state in the $\mu_{\text {surf }} \mathrm{PT}$ simulations. The factor $\mathrm{W}_{\mathrm{id} \text {.gas }}^{\mathrm{ext}}$ is therefore contained in both factors of eq 11 and cancels out in the calculation of the bulk concentration.

2.5. Simulation Details. The inhomogeneous system consisted of between 2000 and 4000 water particles, 4000 oil particles, and a constant box area of $11 \times 11$. Periodic boundary conditions were applied in all three directions. A normal pressure of 23.64 , corresponding toa bulk density of 3.0, was applied by varying the box length.

To study surfactant efficiency at the interface, we do not need to simulate the homogeneous system. As $\mu \mathrm{PT}$ simulations are computationally more expensive than NPT simulations, some of these simulations were performed with a constant number of surfactants (between 40 and 160).

Figure 5a shows a typical snapshot of the simulation box with $120 \mathrm{ht}_{4}$ surfactants. The averagedensity profiles of each bead type are shown in Figure 5b. From both snapshot and density profile, we can clearly see that the surfactants are located mainly at the interface and with the head groups in the water phase and the tail groups in the oil phase. In Figure 5c, the density profile of only the head group is shown at a different scale. From Figure $5 c$, it is evident that not all surfactants stay at theinterface during the entire simulation. The small peaks at $z=19$ and $z=22$ indicate that some form inverse micelles in the oil phase, of which one can be seen in the snapshot in 
(a)

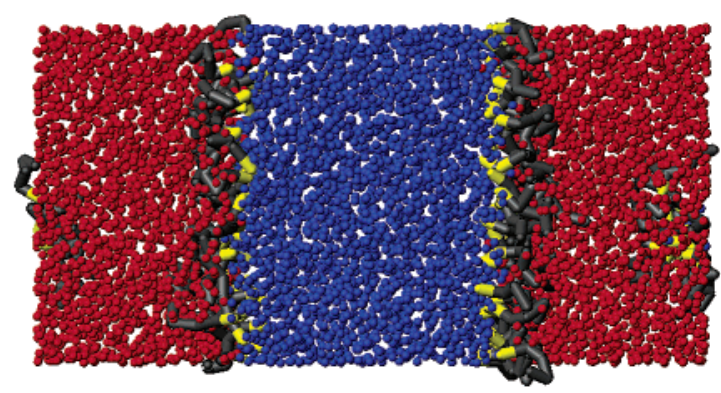

(b)

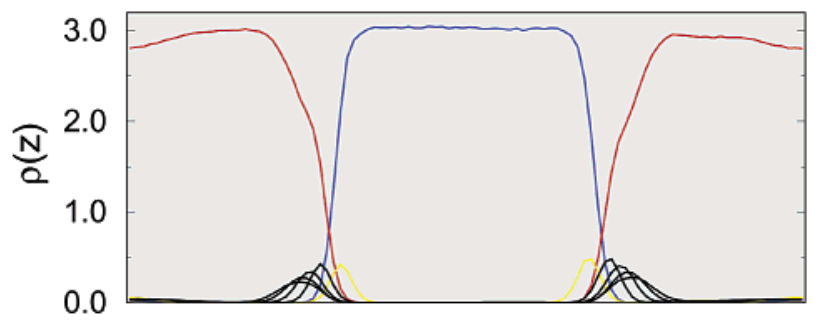

(c)

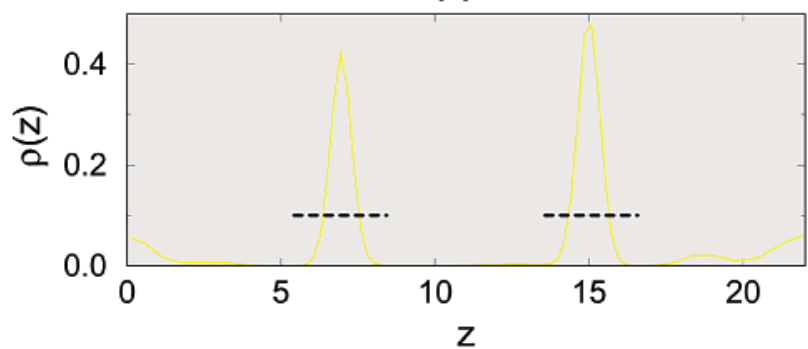

Figure 5. A snapshot of the simulation of oil, water, and the $\mathrm{ht}_{4}$ surfactants (top), the aver age density profiles (middle), and the average density profile of the head groups only (bottom). Oil beads are shown in red, water beads in blue, head groups in yellow, and tail groups in gray.

Figure $5 \mathrm{a}$ (note the periodic boundary conditions). The presence of surfactants in the oil phase explains the low oil density compared to the density of bulk water. Because the size of the inverse micelle is comparable to that of the oil phase, there is a minimum in the middle of the oil phase. We determine the extension of the interface by inspection of the density profile of the head groups (indicated by theblack dashed lines). Theaveragenumber of surfactants at theinterfaceis determined by integrating theaveragedensity profiles over theinterface. This differs from the surface excess (eq 18) when the bulk concentration becomes significant.

In the $\mu_{\text {surf }} \mathrm{P}_{\perp} \mathrm{T}$ simulations, surfactants were inserted and removed using CBMC and 15 trial positions were chosen for each monomer. In all cases, the head group was inserted as the first monomer. After equilibration, the interfacial tension was sampled 800 times during 80000 steps. E ach step invol ves either a series of between 1 and 200 DPD steps, or an attempt to change the box length, or an attempt to insert/remove surfactants. We chose the probability of the first to be 0.5 and that of the latter two to be 0.25 . A time step of 0.03 units was used for the DPD simulations.

For most of thesimulations presented in this work, the bulk concentrations corresponding to low interfacial tension are in the Henry regime. cbulk was therefore estimated from $\mu_{\text {surf }}^{\mathrm{ex}}$ at infinite dilution and Henry's law (eq 11). These calculations were performed in a system of
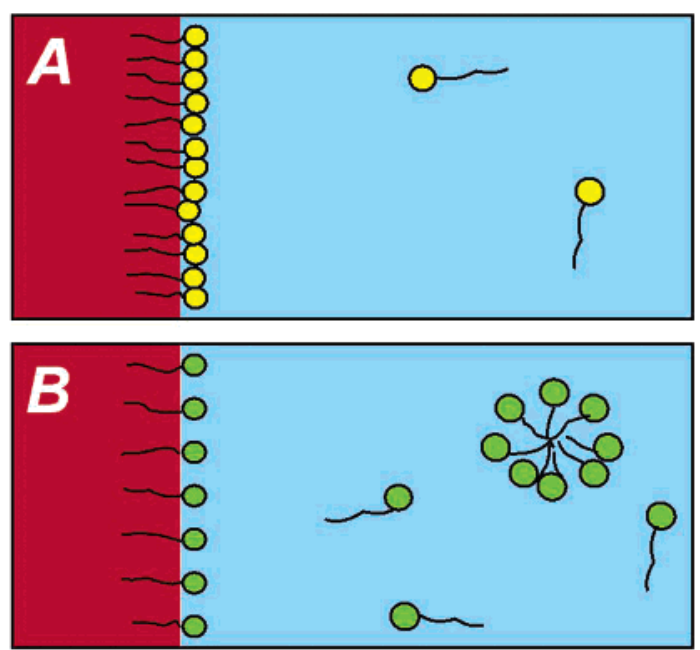

Figure 6. Cartoon of a situation in which the same interfacial tensi on reduction can be obtained with two different surfactant types: A surfactants adsorb more easily at the interface (top), but $\mathbf{B}$ surfactants yield the same interfacial tension because they are more efficient at the interface (bottom).

4000 water particles, and an imposed pressure of 23.64 was applied in all three directions. Sampling of the chemi cal potential is computationally very expensive. We performed 80 trial insertions per Monte Carlo step for more than $10^{6}$ steps, where the step consists of either someDPD steps (on average 100) or an attempt to change the volume.

\section{Results and Discussion}

A surfactant is more efficient than another if fewer surfactant molecules are needed to obtain a given reduction of theinterfacial tension. Experimental ly, interfacial tension can bemeasured using for exampl ea fil m balance (Langmuir trough) or the Wilhelmy slide method. ${ }^{21}$ However, it is difficult to estimate the partition of the surfactants between the bulk and the interface. Possible methods include el lipsometry, neutron scattering, or the use of radioactivetracers. Thefilm balance is mostly used to study monolayers of poorly soluble molecules, and the molecules in the bulk are usually neglected when the interfacial density is estimated. In studies of surfactant efficiency, experiments of theWilhel my slidetypeareoften conducted and the bulk concentration is determined from the total amount of surfactants added. Especially if the second phase is oil rather than air, the surfactant concentration in water at equilibrium can differ significantly from that of the initial solution. M oreover, curves of $\gamma$ as a function of bulk concentration can give different indications than curves of $\gamma$ as a function of interfacial density. Figure 6 shows a hypothetical example: Onecan imagine two systems with different types of surfactants $\mathbf{A}$ and $\mathbf{B}$, but with the sameinterfacial tension reduction. $\mathbf{A}$ has a stronger preference for the interface compared to the bulk than B, but a smaller number of B surfactants at the interface can give the same effect. Comparison of plots of $\gamma$ versus bulk concentration for the two systems would indicate that $\mathbf{A}$ is more efficient, whereas comparison of $\gamma$ versus interfacial density curves would show that at the interface, $\mathbf{B}$ is the better surfactant. In the following sections, we study both of these effects separately. In section 3.1, we investigate the efficiency at the interface for some model surfactants, and in section 3.2,

(21) Adamson, A. W. Physical Chemistry of Surfaces, 5th ed.; Wiley: New York, 1990 


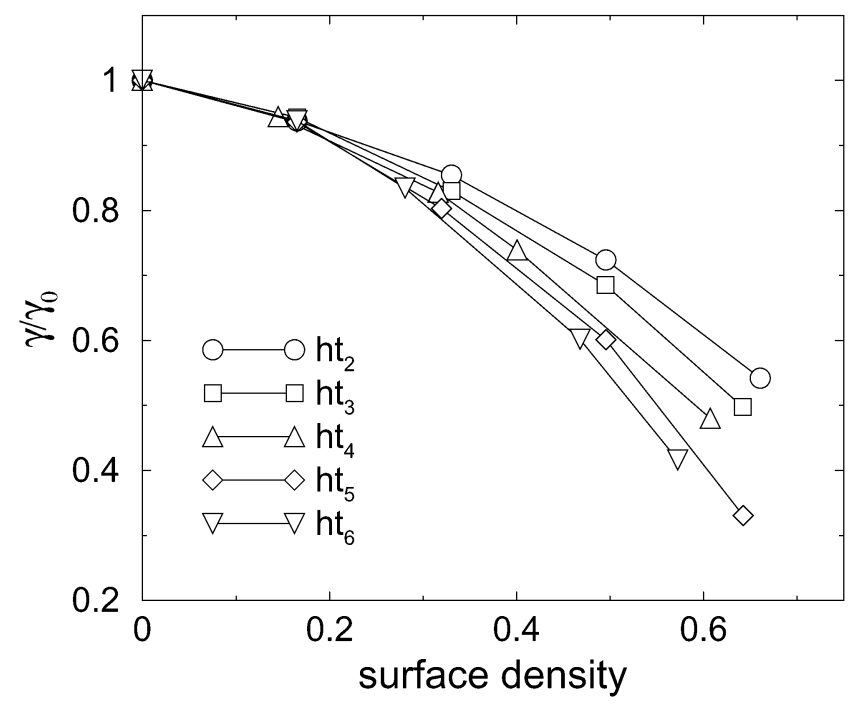

Figure 7. Normalized interfacial tension versus number of surfactants at the interface per area for linear surfactants.

we determine the bulk concentration required to produce a given interfacial tension reduction.

3.1. Surfactant Efficiency at the Interface. In this section, we investigate the effects of changes in the chemical structure on the efficiency at the interface. In particular, weinvestigatetheinfluence of tail length, head group size, topology, and some model parameters.

3.1.1. Influence of Surfactant Size. Figure 7 shows the reduction of theinterfacial tension versus surfactant density at the interface for linear surfactants with the length of the hydrophobic tail increasing from two to six beads. It shows that increasing the tail length enhances the interfacial efficiency of a surfactant. This is in agreement with the well-established Traube's rule and both experiments ${ }^{1}$ and molecular simulations. ${ }^{3}$

If we assume that the monolayer behaves as a twodimensional gas, thefindings can beinter preted in terms of the chain-chain interactions. ${ }^{22}$ Theinterfacial tension reduction $\gamma_{0}-\gamma$ is the two-dimensional analogy to the pressurein threedimensions. For lowinterfacial densities, there is little interaction between surfactants and the surface pressure is low and approximately the same for all surfactant types, corresponding tothetwo-dimensional ideal gas limit. At higher surfactant densities, theexcluded volume interactions and lower conformational entropy yield a higher effective repulsion between surfactants, leading toa higher lateral pressure. This model illustrates why changes in the chemical structure which increase the effective repulsion between surfactants will improve their efficiency.

Figure 8 shows the effect of larger head groups on the interfacial tension. Increasing the size of thehead groups also implies increased effective repulsion between surfactants, and these results can be understood with the same model. Also for surfactants with two hydrophobic tails, an increase in head group or tail length was found to enhance the efficiency.

3.1.2. Effect of the Repulsion Parameters. In our model, we can mimic variation in surfactant properties such as ionic head groups, screening by the addition of salt, and degree of hydrophilic and li pophilic strength by varying the head-head, head-water, and oil-head repulsion parameters. Decreasing the repulsion between head groups corresponds, for example, to adding salt to

(22) Szleifer, I.; Ben-Shaul, A.; Gelbart, W. M. J . Phys. Chem. 1990, $94,5081-5089$.

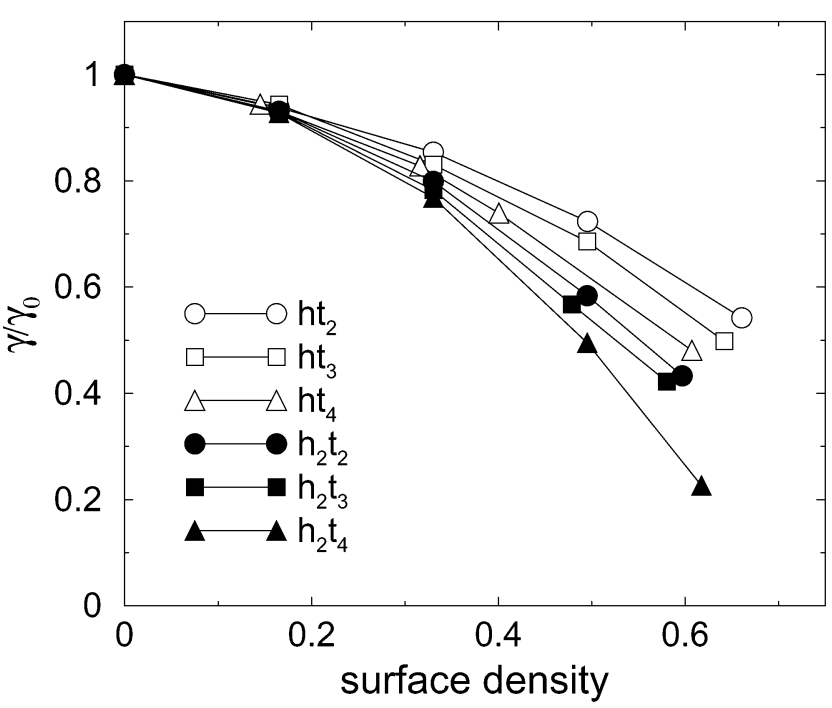

Figure 8. Normalized interfacial tension versus number of surfactants at theinterfaceper area for somelinear surfactants with one (open symbols) and two (filled symbols) head groups.

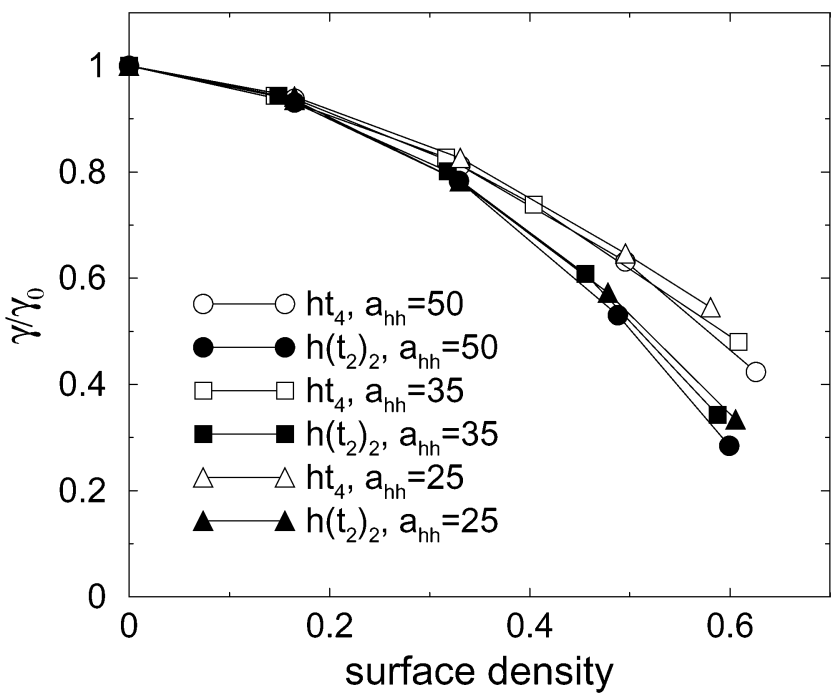

Figure 9. Normalized interfacial tension versus number of surfactants at the interface per area for linear (open symbols) and branched (filled symbols) surfactants. The head-head repulsion parameter $a_{\text {hh }}$ is varied; the other repulsion parameters are as in Table 1.

thesolution. Figure 9 shows the effect of increased headhead repul sion on theinterfacial tension. Thereisa (small) increase in efficiency at the interface, as expected and in agreement with surface pressure-area experiments by Chattopadhyay et al. ${ }^{23}$

Figure 10 shows that decreasing the head-water repulsion parameters has a similar effect. In this system, the tendency for the head groups to be surrounded by water rather than other head groups is enhanced, thus lowering the effective repulsi on between surfactants and decreasing their interfacial efficiency.

A decrease in the oil-head repulsion increases the oil solubility of the surfactant. Figure 11 shows that this has a negative effect on the efficiency. Investigations of the density profiles show that thesurfactants with lower oilhead repul si on stagger more at the interface, as demonstrated by the broader distribution of the surfactants at

(23) Chattopadhyay, A. K.; Ghaïcha, L.; Oh, S. G.; Shah, D. O. J . Phys. Chem. 1992, 96, 6509-6513. 


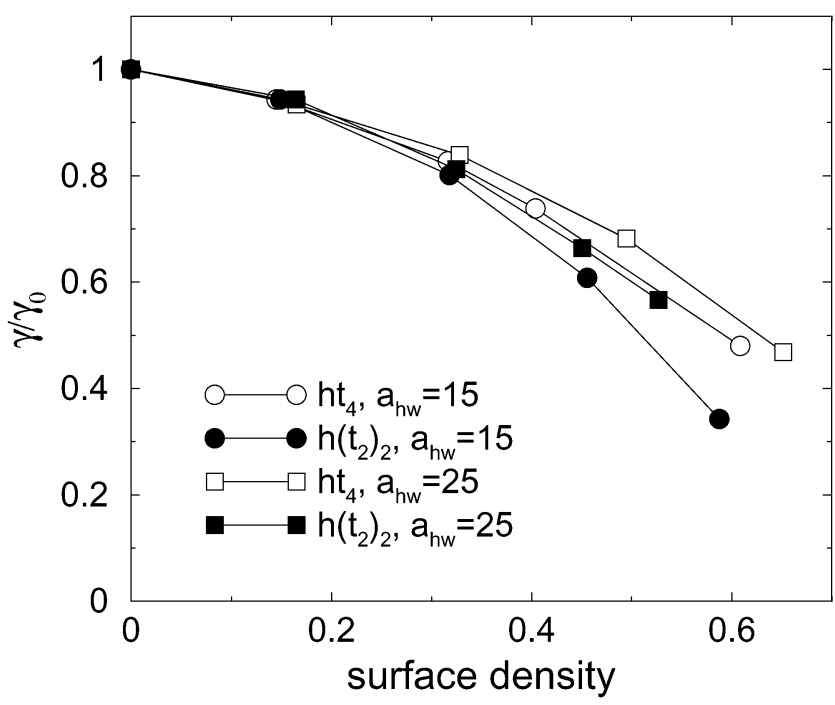

Figure 10. Normalized interfacial tension versus number of surfactants at the interface per area for linear (open symbols) and branched (filled symbols) surfactants. The head-water repulsion parameter $a_{\mathrm{h}}$ is varied; the other repulsion parameters are as in Table 1.

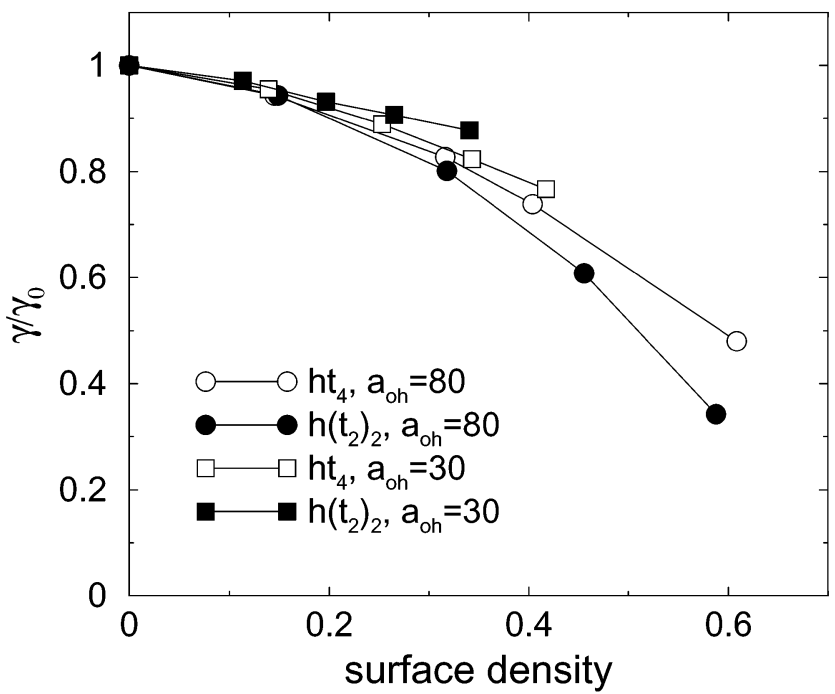

Figure 11. Normalized interfacial tension versus number of surfactants at the interface per area for linear (open symbols) and branched (filled symbols) surfactants. The oil-head (三head-tail) repulsion parameter $a_{\text {on }}$ is varied; the other repulsion parameters are as in Table 1.

the interface in F igure 12a. By such an arrangement, the effectivelateral repulsion decreases, yiel ding lower surface pressure and efficiency. We stress that in the surfactant density on the x-axis in F igure 11, wehave not taken into account thesurfactants in thebulk phases. Thestaggered surfactants are still at the interface, only arranged in a way which reduces the interfacial tension less than those that are more aligned.

3.1.3. Effect of Branching. While the effects of variations in chain length have been known for a long time, the effect of branching the hydrophobic chain is not so well understood. For the repulsion parameters from Groot $^{16}$ (Table 1), branched surfactants are significantly moreefficient than linear ones, seeF igure 11. For another set of repulsion parameters taken from Groot and Rabone, ${ }^{24}$ however, we observed no difference in interfacial

(24) Groot, R. D.; Rabone, K. Biophys. J . 2001, 81, 725-736. (a)

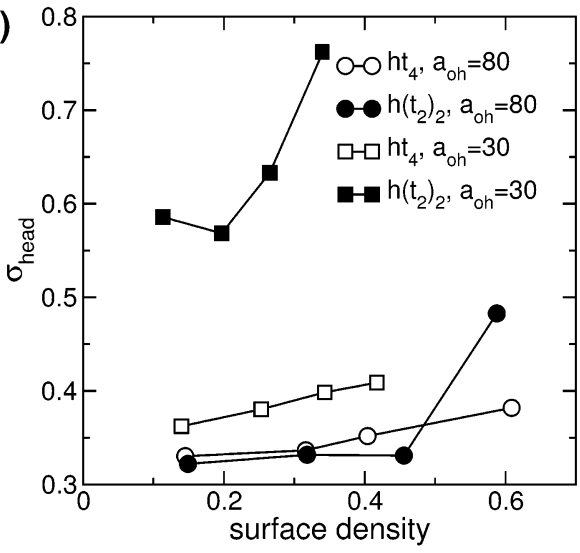

(b)

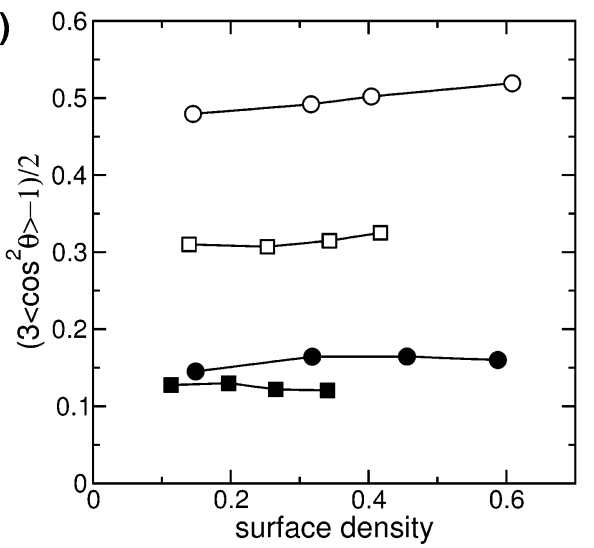

Figure 12. (a) Characteristic width of the head group distribution at the interface, $\sigma_{\text {head. }}$ The oil-head (三head-tail) repulsion parameter $a_{\text {on }}$ is varied; the other repulsion parameters are as in Table 1. (b) The order parameter $\left(3 \cos ^{2} \theta-1\right) / 2$ where $\theta$ is theangle between the bond between the head group and the tail group and the normal to the interface. ( $F$ or the branched surfactants, therearetwosuch bonds, which naturally limits the attainable order.)

Table 1. Repulsion Parameters $a_{i j}$ from Groot, ${ }^{a}$ Except That We Have Used $a_{o o}=25$ Rather Than Their Value of $15^{\mathrm{b}}$

\begin{tabular}{cccc}
\hline & w & o & h \\
\hline w & 25 & 80 & 15 \\
o & 80 & 25 & 80 \\
h & 15 & 80 & 35
\end{tabular}

a Reference 16. ${ }^{\mathrm{b}} \mathrm{w}=$ water bead, $\mathrm{o}=$ oil or tail group, and $\mathrm{h}=$ head group.

efficiency between thelinear and the branched surfactants containing the same number of beads. Systematic variation of the repulsion parameters revealed that the necessary condition for a branched surfactant to be more efficient than its linear isomer is a sufficiently high oilhead repulsion parameter (Figure 11). In other words, branched surfactants are efficient if their head groups are strongly hydrophilic, whereas branched surfactants with less hydrophilic head groups are the least efficient and significantly worse than their linear counterparts.

Astheeffect of theoil-head repulsion on theinteraction between surfactants is not obvious, we investigated the structureand alignment of thesurfactants at theinterface. The density profiles indicated that the distribution of the branched surfactants with low oil-head repulsion, that is, the least efficient ones, is significantly broader than the rest. Figure $12 a$ shows the width of the distribution versus surfactant density at the interface for the linear and branched surfactants with low and high oil-head repulsion. Thesurfactants with lower oil-head repulsion 

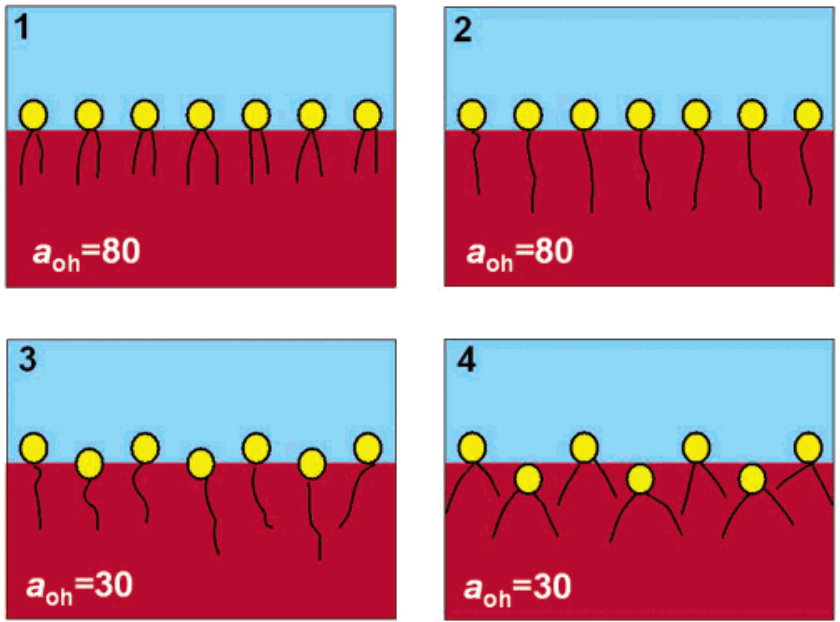

Figure 13. Cartoon of the packing of linear and branched surfactants at theinterfacefor high and low oil-head repulsion parameters. The pictures are numbered in order of decreasing efficiency.

have a broader distribution. This is the result of the molecules staggering at the interface. Moreover, this is much more pronounced for the branched molecules than the linear ones. Figure 12b shows a decrease in the order parameter of the bond(s) between head and tail group when the head group becomes more oil soluble.

It is known that branched mol ecules pack in a different way than linear ones. ${ }^{25,26} \mathrm{However}$, a molecular description of their tension-reducing properties has, to the best of our knowl edge, been lacking. Based on thesimulation results, wecan draw a qualitative picture of theinterfacial packing (Figure 13).

The branched surfactants with less hydrophilic head groups are more efficient than the linear ones because they require more space and have stronger excluded volume interactions. Their strongly hydrophilic head groups force them into a high-entropy arrangement. In this situation, it becomes more beneficial to increase the interfacial area, leading ultimately to a low interfacial tension. However, the branched molecules with more oilsoluble head groups stagger morethan the corresponding linear ones such that they yield lower lateral pressure than the linear ones.

It is interesting tocomparetheseresults with theoretical calculations. In mean-field theories, it is usually assumed that the head groups are fixed in the plane of the interface. ${ }^{22,27}$ Hence, these theories would not allow surfactants to adopt a staggered conformation, and our results suggest that this assumption might lead to an overestimation of the efficiency of branched surfactants.

In the simulations reported here, the tail beads and oil beads have identical properties. However, we also performed simulations in which we lowered the oil- head and head-tail repulsion independently. This showed that indeed the oil-head repulsion was crucial for obtaining linear surfactants that weremore efficient at theinterface than the branched ones. The same results were observed for longer surfactants ( $h t_{6}$ compared to $\left.h\left(t_{3}\right)_{2}\right)$ and for oil chains of two and three oil beads.

(25) Ghaïcha, L.; Leblanc, R. M.; Chattopadhyay, A. K.J . Phys. Chem. 1992, 96, 10948-10953.

(26) Green, S. R.; Su, T. J .; Lu, J . R.; Penfold, J J J . Phys. Chem. B 2000, 104, 1507-1515.

(27) van Os, N. M.; Rupert, L. A. M.; Smit, B.; Hilbers, P. A. J .; Esselink, K.; Böhmer, M. R.; Koopal, L. K. Colloids Surf., A 1993, 81, 217-229.

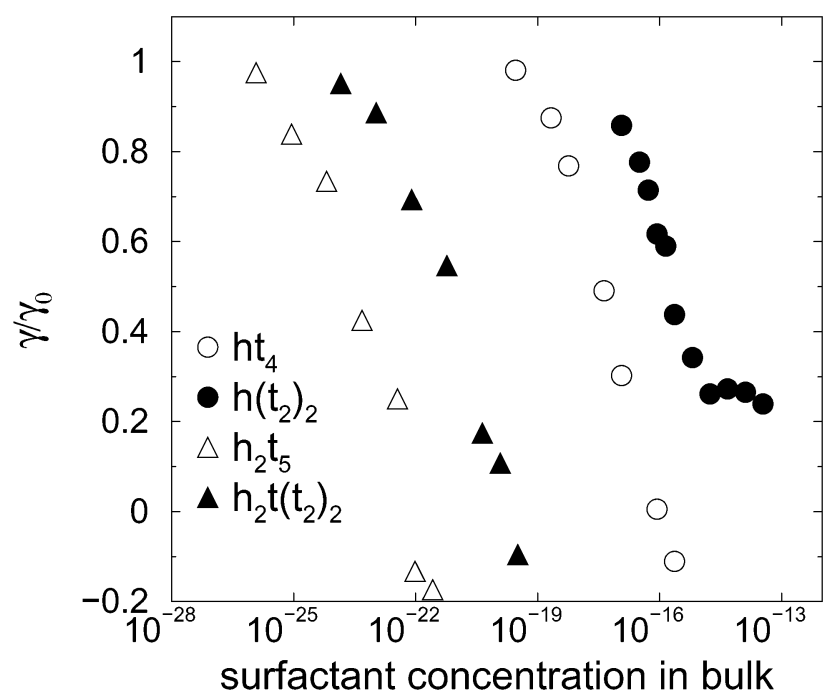

Figure 14. I nterfacial tension versus surfactant concentration in the bulk for linear surfactants (open symbols) and branched surfactants (filled symbols). They-error bars areabout thesize of the symbols. An order of magnitude error in the Henry coefficients implies an uncertainty of an order of magnitude in the bulk concentration for each curve.

3.2. Interfacial Tension versus Bulk Concentration. The overall efficiency of a surfactant depends on two factors: the tendency to adsorb on the interface and its efficiency at the interface. By using the method described in sections 2.3-2.4, we determined the bulk surfactant concentration required to produce a given interfacial tension. Simulations were performed with four different surfactants: the linear $\mathrm{ht}_{4}$, its branched isomer $h\left(t_{2}\right)_{2}$, and two larger surfactants, $h_{2} t_{5}$ and $h_{2} t\left(t_{2}\right)_{2}$. The two tails of $h_{2} t\left(t_{2}\right)_{2}$ are connected to a hydrophobic rather than a hydrophilic bead, to resembleGuerbet surfactants, see Figure 1.

The reduction of the interfacial tension versus bulk concentration is shown in Figure 14. For thesesurfactants, the bulk concentrations remain in the Henry regimeeven for imposed chemical potentials corresponding tovery low interfacial tension. The bulk concentration as a function of surfactant chemical potential can thereforebeestimated from Henry's law.

Wefound littl edifferencein $\mu_{\text {surf }}^{\mathrm{ex}}$ between $\mathrm{ht}_{4}$ and $\mathrm{h}\left(\mathrm{t}_{2}\right)_{2}$. The Henry coefficients were $8 \times 10^{-46}$ and $2 \times 10^{-45}$, respectively. F or theseven-beaded surfactants, theH enry coefficient is 2 orders of magnitude higher for the linear than the branched one: $4 \times 10^{-62}$ compared to $4 \times 10^{-64}$. Nevertheless, much higher bulk concentrations of the branched isomers are needed to obtain thesameinterfacial tension compared to the linear ones. This is because, to obtain a certain interfacial tension, a higher chemical potential must be imposed in the inhomogeneous system for branched surfactants: the factor $\exp \left\{-\beta\left(\mu_{\text {surf }}-\right.\right.$ $\left.\mu_{\text {idgas }}^{0}\right\}$ at the interface is about 4 orders of magnitude lower for $h_{2} t\left(t_{2}\right)_{2}$ compared to $h_{2} t_{5}$. This shows that the same reasons that make branched surfactants more efficient at theinterfacemakethem less inclined toadsorb at the interface.

Some of thesurfactants areabletoreducetheinterfacial tension tozero. At the corresponding chemical potentials, we do not observe surfactants in the water system; that is, no insertions are accepted in the Monte Carlo scheme. We performed several simulations starting with surfactants and micelles in water and found that significantly higher chemical potentials must be applied in order to 
(a)

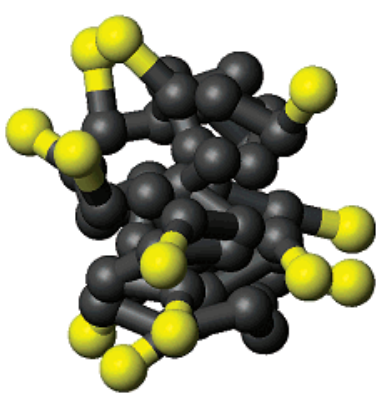

(b)

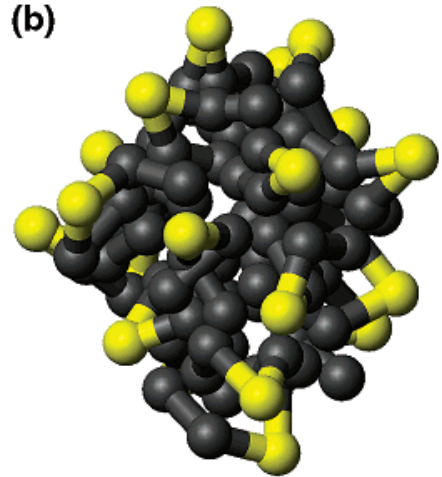

Figure 15. Micelles in the water phase of the homogeneous system. Head groups are shown in yellow, and tail groups in gray. For clarity, water particles are not shown. (a) 12 ht $_{4}$ surfactants. (b) $20 \mathrm{~h}\left(\mathrm{t}_{2}\right)_{2}$ surfactants.

(a)

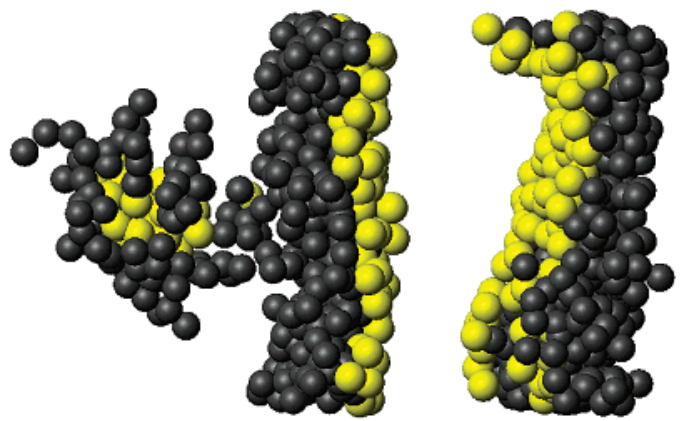

(b)
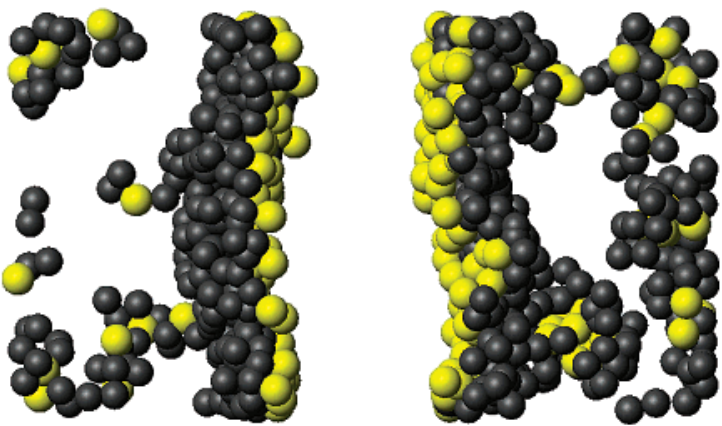

Figure 16. Snapshots of the inhomogeneous simulation systems. Head groups are in yellow, and tail groups in gray. For clarity, water and oil particles are not shown. (a) ht $_{4}$ surfactants at $\gamma=0$. (b) $h\left(t_{2}\right)_{2}$ surfactants at $\gamma=0.25 \gamma_{0}$.

obtain stablemicelles. Somesnapshots of theseareshown in Figure 15.

Because the lipophilic parts of the $h t_{4}$ and $h\left(t_{2}\right)_{2}$ surfactants are more dominating than the hydrophilic parts, it is not surprising that the surfactant concentration in theoil phaseis considerableat high chemical potentials. We observe both single surfactants and aggregates. For the linear molecules, the concentration at the interface continues to increase with higher chemical potential together with increasing concentration in the oil phase. Figure 16a shows a snapshot with a curved interface and an inverse micelle in the oil phase, from the simulation of $\mathrm{ht}_{4}$ which gave $\gamma=0$.

The $\gamma\left(c_{\text {bulk }}\right)$ curves for $h\left(t_{2}\right)_{2}$, however, flatten out at a certain chemical potential. Since this is still in the Henry regime in the water phase, it does not indicatethecritical micelle concentration in water. Rather it reflects that surfactants start to aggregatein theoil phase. A snapshot of this is shown in Figure 16b. We can therefore from Figure 14 locate the surfactant concentration in water that corresponds to thecritical aggregation concentration

in the oil. The inverse micelles are observed in the inhomogeneous system. As the system is relatively small and dominated by the interface, the surfactant concentration in theoil phasecannot be determined very accurately. This could be solved by performing the same $\mu_{\text {surf }} \mathrm{PT}$ simulations in a bulk oil system.

The negative interfacial tension values in Figure 14 indicate a metastable system. We suggest that this is a finite-size effect because the size of (inverse) micelles is comparable to the size of the bulk phases in the inhomogeneous simulation box. Although we did not observe stablemicelles in bulk water at thesechemical potentials, we expect inverse micelles in oil. One inverse micelle is indeed observed in the inhomogeneous system (Figure 16a).

The low Henry coefficients in water are partly due to the domi nating hydrophobic tails. In oil we found Henry coefficients $10^{14}-10^{17}$ times larger than in water. Themain reason for such low solubility is the large hydrophilichydrophobic repulsion (80 compared to $\mathrm{a}_{w w}=25$ ). To convert the concentrations in Figure 14 to $\mathrm{mol} / \mathrm{dm}^{3}$, the reduced units must be multiplied by a factor of 40 if we map three $\mathrm{CH}_{2}$ groups onto one bead. These values are very low compared to the experimental values. ${ }^{4} \mathrm{~A}$ lower hydrophilic-hydrophobic repulsi on parameter compared to that of water-water, such as in ref 24 , will reproduce the solubility better.

Our simulations show that lower bulk concentrations of linear surfactants than branched ones are needed to obtain the same interfacial tensi on reduction. This is in agreement with the results of Aspée and Lissi, ${ }^{7}$ who concluded that when the surface coverage is high enough for steric repulsion to be important, branched isomers of nonanol will have a higher free energy at the interface. Our results agree also qualitatively with experimental work on Guerbet branched surfactants with ionic or nonionic head groups. ${ }^{6}$ Our results indicate that, given the ingredients of the model, one cannot design a surfactant for which the branched isomer gives the lowest $\gamma\left(c_{\text {bulk }}\right)$ curves, although this is reported in theliterature ${ }^{4,5}$ This might be a limitation of the simple model we have used. However, our results do agree with the majority of the experimental record. ${ }^{1}$ We also note that the experimental conditions in refs 4 and 5 are not directly comparableto our simulations. Varadaraj et al. measured instantaneous interfacial tension values on nonequilibrated samples using the spinning drop technique. ${ }^{4}$ Pitt et al. compared surfactants that have the same critical aggregation concentration, rather than the samenumber of methylene groups. ${ }^{5}$

\section{Conclusion}

We have shown that DPD simulations of simple oil/ water/surfactant systems can predict surfactant behavior at interfaces. The higher efficiency at the interface for increasing chain lengths and larger or more ionic head groups is in agreement with the literature. Our simulations reveal that branching has a positive effect on the efficiency at the interface only if the head group is hydrophilic enough to maintain a compact layer despite the steric repulsion between the chains.

Furthermore, we have shown a method to determine thebulk concentrations in equili brium with theinterface. For the model surfactants investigated, higher bulk concentrations of branched isomers are required to reduce the interfacial tension by a given amount, compared to thelinear ones. Wefound that thesamereasons that make branched surfactants efficient at theinterfacemakethem 
less inclined toadsorb at theinterface. Although our model is simple and only a limited number of surfactant models havebeen investigated, wecould point out themechanisms and surfactant properties which determine surfactant efficiency at the interface and tendency to adsorb at the interface. Our findings do not suggest any branched surfactants to be more efficient than their linear isomers at concentrations in the Henry regime.
Acknowledgment. These investigations are supported in part by the N orwegian Research Council (Grant No. 145184/432), by The Netherlands Research Council for Chemical Sciences (CW), and by The Netherlands Organization for Scientific Research (NWO) through PIONER.

LA0346346 